\title{
CrystEngComm
}

Check for updates

Cite this: CrystEngComm, 2021, 23, 5815

Received 13th May 2021,

Accepted 27th July 2021

DOI: $10.1039 / \mathrm{d} 1 \mathrm{ce} 00642 \mathrm{~h}$

rsc.li/crystengcomm

\section{Tuning the mechanical flexibility of organic molecular crystals by polymorphism for flexible optical waveguides $\dagger$}

\author{
Torvid Feiler, (D) ab Biswajit Bhattacharya, (D)*a Adam A. L. Michalchuk, (D) ${ }^{a}$ \\ Seon-Young Rhim, (iD c Vincent Schröder, ${ }^{\text {cd }}$ \\ Emil List-Kratochvil iD *cd and Franziska Emmerling iD *ab
}

\begin{abstract}
The ability to selectively tune the optical and the mechanical properties of organic molecular crystals offers a promising approach towards developing flexible optical devices. These functional properties are sensitive to crystallographic packing features and are hence expected to vary with polymorphic modification. Using as a model system the photoluminescent material 4-bromo-6-[(6-chloropyridin-2-ylimino)methyl]phenol (CPMBP), we herein demonstrate the simultaneous tuning of mechanical flexibility and photoluminescence properties via polymorphism. Two new polymorphic forms of CPMBP were obtained from a solution and fully characterised using a combination of experiments and density functional theory simulations. These polymorphic forms exhibit remarkably distinct mechanical properties and an order of magnitude difference in photoluminescence quantum yield. The mechanically plastic form has a higher quantum yield than the brittle polymorphic form. However, their photoluminescence emission profile is largely unaffected by the observed polymorphism, thereby demonstrating that the optical properties and bulk mechanical properties can in principle be tuned independently. By distinguishing between active (involving absorption and emission) and passive (involving no absorption) light propagation, the waveguiding properties of the plastic form of CPMBP (form II) were explored using the straight and bent crystals to highlight the potential applications of CPMBP in designing flexible optical devices. Our results demonstrated that polymorph engineering would be a promising avenue to achieve concurrent modulation of the optical and mechanical properties of photoluminescent molecular crystals for next-generation flexible optical device applications.
\end{abstract}

\section{Introduction}

Soft amorphous materials such as organic polymers and elastomers have been studied extensively in academia and industry due to their high degree of mechanical adaptivity. ${ }^{1-5}$ In contrast, crystalline molecular materials are often brittle and prone to breaking upon external mechanical forces. This fragility of crystalline materials poses challenges for their applications in next-generation technologies, including as

${ }^{a}$ BAM Federal Institute for Materials Research and Testing, Richard-Willstätter-Str. 11, 12489 Berlin, Germany. E-mail: biswajit.bhattacharya@bam.de,

franziska.emmerling@bam.de

${ }^{b}$ Department of Chemistry, Humboldt-Universität zu Berlin, Brook-Taylor-Str. 2, 12489 Berlin, Germany

${ }^{c}$ Department of Chemistry, Department of Physics, Humboldt-Universität zu Berlin, IRIS Adlershof, Zum Großen Windkanal 2, 12489 Berlin, Germany.

E-mail: emil.list-kratochvil@hu-berlin.de

${ }^{d}$ Helmholtz-Zentrum Berlin für Materialien und Energie GmbH, Hahn-MeitnerPlatz 1, 14109, Berlin, Germany

$\dagger$ Electronic supplementary information (ESI) available. CCDC 2082067 and 2082068. For ESI and crystallographic data in CIF or other electronic format see DOI: $10.1039 / \mathrm{d} 1 \mathrm{ce} 00642 \mathrm{~h}$ sensors, synthetic tissues, and advanced optoelectronics. Correspondingly, the recent discovery of mechanical flexibility in single crystals ${ }^{6}$ of molecular materials ${ }^{7-16}$ and coordination polymers $^{17-20}$ opens new opportunities to design nextgeneration flexible technologies. Numerous applications of flexible molecular crystals have been already demonstrated including their use as optical waveguides, ${ }^{21-26}$ microoptoelectronic devices, ${ }^{27,28}$ sensors, ${ }^{29,30}$ and biomimetics. ${ }^{31,32}$ While a growing number of mechanically flexible crystalline molecular materials are being reported, they remain scarce and their preparation is largely serendipitous.

Based on their qualitative mechanical behavior, the flexibility of molecular crystals can be classified as plastically (irreversible) and elastically (reversible) bendable. Plastic bending in molecular crystals has been rationalized based on anisotropic molecular arrangements and the existence of facile slip planes where molecular layers interact via weak dispersive intermolecular interactions such as halogen bonding, $\pi \cdots \pi$ stacking, or weak van der Waals interactions. $^{7,15}$ In contrast, elastic bending arises from energetically isotropic molecular packing with weak 
interactions. $^{9,33}$ These weak interactions facilitate the displacement of molecules without disrupting the stabilizing forces that maintain the structural integrity of the crystal. The success of these structural criteria for elastic and plastic bending demonstrates that the bulk mechanical compliance of molecular materials is rooted in their crystal packing and non-covalent interactions (NCIs).

The most obvious way to tune NCIs in crystalline materials is to modify the chemical functionality. This approach has been used widely in molecular and coordination polymers for altering the mechanical compliance..$^{7,18,34}$ However, this approach is drastic and can change significantly the critical functional properties of the molecules. To overcome this limitation, crystal engineering strategies have been developed which allow NCIs to be selectively tuned by introducing multiple components in the crystal packing, cocrystallisation and salt formations. ${ }^{35-38}$ For example, Nath et al. recently reported how the plasticity of probenecid crystals could be infused into brittle 4,4'-bipyridine via cocrystallisation. ${ }^{35}$ Although this crystal engineering approach does not require modification of the chemical functionality, the inclusion of multiple chemical entities into the material can impose restrictions on material compatibility. For example, where the material application requires biological or environmental compatibility, the inclusion of a toxic coformer would render the material unusable. ${ }^{39}$ To avoid any chemical modification whatsoever, NCIs can also be tuned by controlling polymorphism. Polymorphism stems from the ability of a molecule to crystallise in more than one solid form, each having distinct arrangements or conformations of the constituent molecules. ${ }^{40}$ Tuning the mechanical flexibility by controlling polymorphism of a molecular crystal of 6-chloro2,4-dinitroaniline was first reported by Reddy and Desiraju in their pioneering work. ${ }^{41}$ More examples of tuning the mechanical response of molecular crystals through polymorph screening have been since reported. ${ }^{42-46}$

The polymorphism of molecular crystals which show simultaneously mechanical flexibility and useful optical properties is rare. Single crystals of $\pi$-conjugated organic chromophores are frequently used as active micro-optical components such as optical waveguides, ${ }^{47}$ lasers, ${ }^{48}$ circuits, ${ }^{49}$ and field-effect transistors $^{50}$ in designing advanced optoelectronic devices. However, these devices are predominantly prepared by using the brittle forms of single crystals. By coupling mechanical flexibility to optical properties, a variety of optoelectronic applications can be envisioned. For example, by making use of elastically flexible materials, reversible optical sensors become feasible, whereas shapable nano-optical devices can be envisioned through the use of plastically bendable crystals.

In recent years, researchers have successfully identified mechanically flexible luminescent molecular materials. For example, significant efforts have been devoted to designing mechanically flexible optical waveguides. ${ }^{20-26}$ These materials have the properties of bending the path of light, thereby offering potential applications in nano-optical devices.
Similarly, various materials have been reported whose optical properties can be tuned by the extent of bending. ${ }^{29,30}$ More recently, advanced optical properties have also been demonstrated to be tunable by mechanical bending including the polarization of plane polarized light. ${ }^{51}$ Thus, fine-tuning the interplay between optical and mechanical properties has significant potential for next-generation functional materials. Only recently has polymorphism been revealed as a potential design strategy for simultaneously modifying both the optical and mechanical properties of molecular crystals. Although the strategy seems promising, only limited number of examples have been reported. Further studies are required to explore the breadth of this phenomenon before its full potential can be realized.

Polymorphism in molecular crystals is ubiquitous. ${ }^{40}$ For the purpose of this study, we opt to explore the polymorphism of 4-bromo-6-[(6-chlorolpyridin-2-ylimino) methyl]phenol (CPMBP). This molecule was selected as salicylaldehyde-derived Schiff bases are well known to be highly photoluminescent and often show polymorphism in the solid state. ${ }^{52}$ Moreover, the chlorine and the bromine atom on the backbone of the molecule can be expected to introduce mechanical compliance in crystal forms. This molecule has significant potential for studying the simultaneous effect of polymorphism on the optical and mechanical properties.

\section{Experimental}

\section{Materials}

2-Amino-6-chloropyridine (98\%; CAS No: 45644-21-1) and 5-bromosalicylaldehyde (98\%; CAS No: 1761-61-1) were purchased from Fluorochem and Alfa Aesar, respectively, and used as received.

\section{Synthesis and crystal growth}

2-((E)-(6-Chloropyridin-2-ylimino)methyl)-4-bromophenol (CPMBP) was synthesised by liquid-assisted mechanochemical grinding (LAG) with methanol $(100 \mu \mathrm{L})$ of an equimolecular mixture of 5-bromosalicylaldehyde (201 $\mathrm{mg}, 1 \mathrm{mmol}$ ) and 2-amino-6-chlropyridine (129 $\mathrm{mg}, 1 \mathrm{mmol}$ ) with a mortar and pestle. Single crystals were grown by dissolving the ground powder in DCM, followed by slow evaporation or anti-solvent crystallization in a beaker or tube.

\section{Powder X-ray diffraction (PXRD)}

The ground crystals were packed into borosilicate capillaries (inner diameter: $0.5 \mathrm{~mm}$ ). PXRD data were collected on a Bruker D8 Discover diffractometer (Bruker AXS, Karlsruhe, Germany) equipped with a LYNXEYE XE detector and using $\mathrm{Cu}-\mathrm{K \alpha}_{1}$ radiation $(\lambda=1.506 \AA)$. Data were collected from $2 \theta=$ $3^{\circ}$ to $50^{\circ}$ with a step size of $0.009^{\circ}$ and $6 \mathrm{~s}$ per step. 


\section{Single-crystal X-ray diffraction}

Single crystal X-ray diffraction (SCXRD) data of both polymorphs were collected using a Bruker D8 Venture diffractometer (Bruker AXS, Karlsruhe, Germany) using graphite-monochromated Mo $\mathrm{K} \alpha$ radiation $(\lambda=0.71073 \AA)$. Data reduction was performed using the Bruker AXS SAINT ${ }^{53}$ and SADABS ${ }^{54}$ software packages. Both structures were solved by SHELXT 2018 (ref. 55) using direct methods, followed by successive Fourier and difference Fourier syntheses. Fullmatrix least-squares refinements were done on $F^{2}$ using SHELXL 2018, ${ }^{56}$ including anisotropic displacement parameters for all non-hydrogen atoms. Hydrogen atoms bonded to oxygen were located from the electron density maps, and all hydrogen atoms bonded directly to carbon atoms were fixed at their ideal positions. Data collection, structure refinement parameters, and crystallographic data for the cocrystals are summarized in Table S1. $\dagger$

\section{Differential scanning calorimetry (DSC)}

Differential scanning calorimetry (DSC) measurements were performed with a TGA/DSC 3+ (Mettler Toledo, Greifensee, Switzerland). The measurements were performed in open aluminum crucibles under a continuous $\mathrm{N}_{2}$-air flow. The experiments were conducted at a heating rate of $10 \mathrm{~K} \mathrm{~min}^{-1}$ over a range of $25^{\circ} \mathrm{C}$ to $550{ }^{\circ} \mathrm{C}$.

\section{Fourier-transform infrared spectroscopy (FTIR)}

FTIR spectra of the powdered samples were measured using a Nicolet FT-IR NEXUS (Thermo Fisher, Dreieich, Germany) spectrometer equipped with a Diamond-ATR-Golden Gate unit and a DTGS KBr detector. Each spectrum was collected in a range of $200-4000 \mathrm{~cm}^{-1}$ with a spectral resolution of 4 $\mathrm{cm}^{-1}$.

\section{Photoluminescence spectroscopy}

Photoluminescence measurements were performed with an Edinburgh Instruments (Livingston, UK) FLS 980 fluorescence spectrometer. All photoluminescence spectra were measured using an integrating sphere. The samples were excited with a $450 \mathrm{~W}$ ozone-free xenon arc lamp. The detector was a R928P PMT, electrically cooled to $-20{ }^{\circ} \mathrm{C}$.

\section{Waveguide}

The use of CPMBP as a waveguide material was tested by using LED light sources (Thorlabs Inc.). For the measurements, the CPMBP crystals were placed on a silicon wafer with a $3 \mu \mathrm{m}$ thick $\mathrm{SiO}_{2}$ layer, and the light was coupled into the crystals with a microscope objective (100× magnification, 0.8 numeric aperture (NA), Nikon Inc.) using the end-fire coupling method. Coupling the light in the direction of the optical axis of the waveguide facilitates the investigation of propagation behavior. At the other end of the crystal, the outcoupled light was collected perpendicular to the optical axis of the waveguide by applying another objective (10× magnification, 0.25 numeric aperture). The collected light was subsequently guided via fibers to a CCD detector (Kymera 328i, Oxford Instruments Inc.). Since CPMBP shows photoluminescence, we differentiate between active and passive waveguiding. Active waveguiding describes the propagation of the emitted light caused by the photoluminescence, providing that the wavelength of the LED source is in the excitation range. The light propagation without any interaction is denoted as passive waveguiding. A schematic picture of the measurement setup can be found in the ESI, $\dagger$ Scheme S1.

\section{Computational methods}

Initial structures were taken from the experimentally determined single crystal X-ray diffraction data, as described above. Plane wave density functional theory (pw-DFT) calculations were performed using Quantum ESPRESSO v6.4. ${ }^{57,58}$ Structure relaxation was performed using the generalized gradient approximation (GGA) exchangecorrelation functional of Perdew-Burke-Ernzerhof $(\mathrm{PBE})^{59}$ with the exchange-dipole moment $(\mathrm{XDM})^{60}$ dispersion correction. The nuclear-electron interactions were approximated using the projector augmented-wave method, ${ }^{61}$ and the electronic wave function was expanded in plane waves to a kinetic energy cut-off of $80 \mathrm{Ry}$. Convergence of the electronic wave function was accepted with change in energy $<10^{-10} \mathrm{Ry}$, and forces were considered to be converged when $<10^{-5}$ Ry a. $\mathrm{u}^{-1}$. The Brillouin zone was sampled on a Monkhorst-Pack grid ${ }^{62}$ with $0.06 \AA^{-1}$ for form I and $0.05 \AA^{-1}$ for form II, respectively.

Intermolecular interaction energies were calculated using the CLP-PIXEL $\operatorname{method}^{63,64}$ and associated wrapper MrPIXEL, ${ }^{65}$ using a condensation level of 4 and a cluster radius of $15 \AA$ A. PIXEL calculations were based on $a b$ initio charge densities (grid size: $0.06 \AA$ ) produced at the B3LYP/6$31 \mathrm{G}(\mathrm{d}, \mathrm{p})$ level of theory with Gaussian v16, ${ }^{66}$ with hydrogen positions normalized to conventional neutron data.

\section{Results and discussion}

The compound, CPMBP, was synthesized by liquid-assisted mechanochemical grinding (LAG) with methanol of an equimolecular mixture of 5-bromosalicylaldehyde and 2-amino-6-chlropyridine, Fig. 1a. Slow evaporation of a saturated $\mathrm{CH}_{2} \mathrm{Cl}_{2}$ solution of CPMBP at room temperature in a beaker yielded flake-shaped orange crystals. When the crystals were handled with a metal needle, some crystals displayed brittle nature and others showed plastic bending and remained deformed even after removal of the stress, Fig. $1 \mathrm{~b}$ and c, suggesting the concomitant formation of two polymorphic forms. In contrast, crystallization of CPMBP in pure DCM or using an anti-solvent in glass tubes produced pure mechanically distinct flake-shaped crystals, presumably owing to the different crystallisation kinetics as compared with crystallisation from a wide-mouthed beaker. Crystals obtained from DCM with $n$-hexane were brittle (form I) over both the long crystallographic faces. In contrast, crystals 
(a)<smiles>Oc1ccc(Br)cc1/C=N/c1cccc(Cl)n1</smiles>

2-((E)-(6-chloropyridin-2-ylimino) methyl)-4-bromophenol (CPMBP)

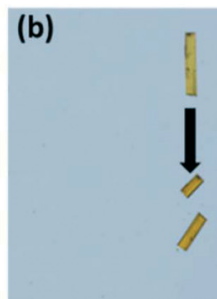

Form I (c)

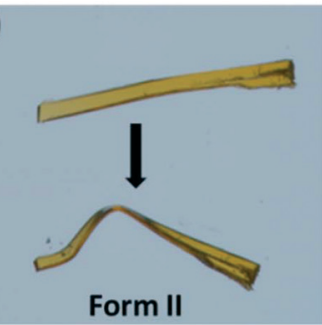

Fig. 1 (a) Chemical structure of the title compound, CPMBP. (b and c) Optical images showing the brittle and plastic deformation behaviour of form I and form II, respectively.

obtained from pure DCM deformed plastically (form II) over the major faces but appeared brittle over the other faces. Powders of each of the pure crystal forms exhibited a single endothermic peak in their differential scanning calorimetric profiles, corresponding to their melting point (Fig. S1†): 153.8 ${ }^{\circ} \mathrm{C}$ and $158.0{ }^{\circ} \mathrm{C}$ for form I and form II, respectively. The phase purity is also consistent with the unique PXRD diffraction pattern of the powders of the crystals (as compared with the simulated PXRD pattern from single crystal data, Fig. S2 $\dagger$ ) and FT-IR spectra (Fig. S3†). The FT-IR spectra of the two polymorphs are nearly identical. For example, the aromatic $\mathrm{C}-\mathrm{Br}$ stretching vibration in both forms is at $1072 \mathrm{~cm}^{-1}$. The $\mathrm{C}-\mathrm{H}$ deformation vibration of the 2,6-substituted pyridines is also at the same position, 792 and $730 \mathrm{~cm}^{-1}$, respectively. ${ }^{67}$
To understand the intermolecular interactions and molecular packing motifs responsible for the observed distinct mechanical flexibility of the two different crystalline forms of CPMBP, the crystal structures of both forms were determined by SCXRD. Form I (brittle) crystallizes in the orthorhombic space group Pca2 $2_{1}$ with one CPMBP molecule in the asymmetric unit. The cell parameters are $a=25.13 \AA$, $b$ $=3.87 \AA$ and $c=11.98 \AA$ (Table S1 $\dagger$ ). The CPMBP molecules adopt a near-planar conformation with a dihedral angle of $8.4^{\circ}$ between the pyridyl and phenolic rings, driven by the intramolecular $\mathrm{O}-\mathrm{H} \cdots \mathrm{N}$ hydrogen bonds (Fig. S4a; $\dagger \mathrm{H} \cdots \mathrm{A}$ : $\left.1.86 \AA, \theta: 146 \cdot 1^{\circ}\right)$. Crystal face indexing by SCXRD indicates that the two long crystallographic faces are the $(001) /(00 \overline{1})$ and $(010) /(0 \overline{1} 0)$ faces, whereas $(100) /(\overline{1} 00)$ are the top faces (a)

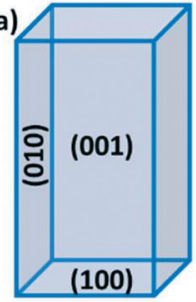

(b)

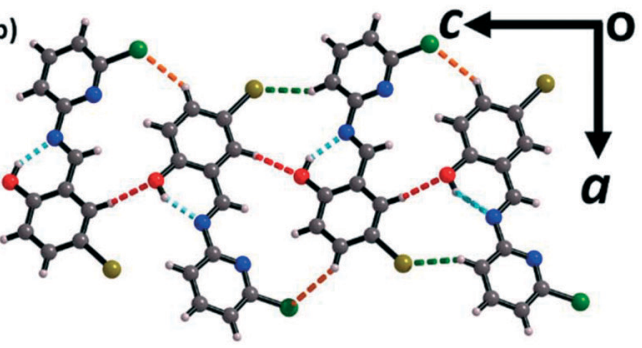

(c)

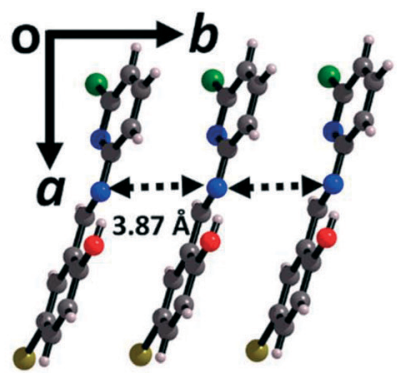

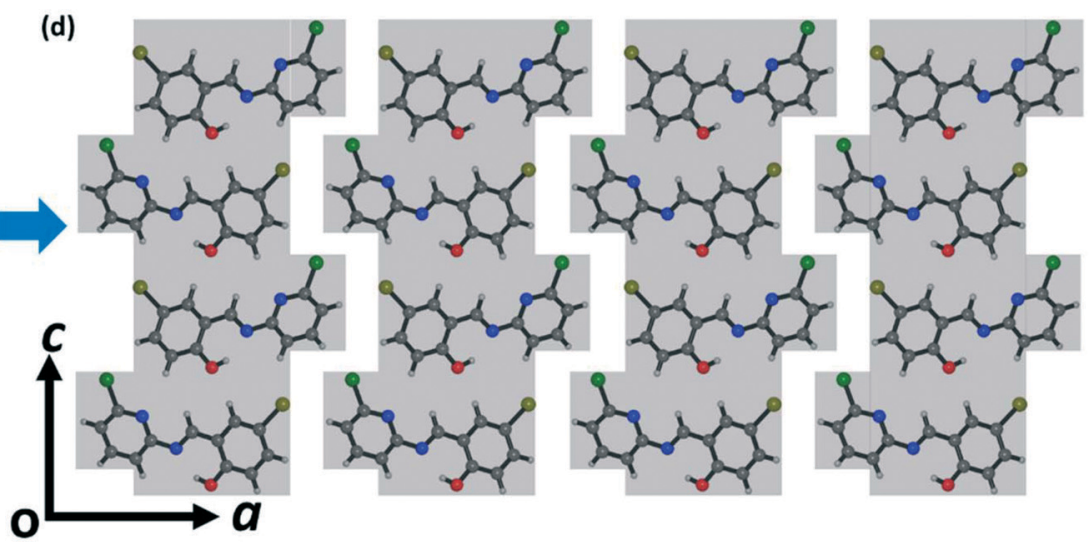

(e)

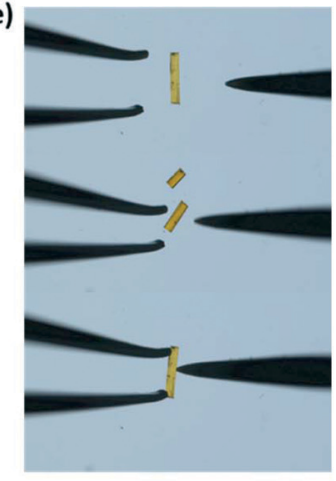

Fig. 2 Molecular packing in the crystal structure of form I of CPMBP. (a) Schematic diagram of the crystal face indices determined by singlecrystal X-ray diffraction. (b) The $1 \mathrm{D}$ ribbon is formed via $\mathrm{C}-\mathrm{H} \cdots \mathrm{O}, \mathrm{C}-\mathrm{H} \cdots \mathrm{Cl}$ and $\mathrm{C}-\mathrm{H} \cdots \mathrm{Br}$ interactions along the $\mathrm{C}$-axis $(\mathrm{C}-\mathrm{H} \cdots \mathrm{O}$ interactions: red dotted lines, $\mathrm{O}-\mathrm{H} \cdots \mathrm{N}$ interactions: cyan dotted lines, $\mathrm{C}-\mathrm{H} \cdots \mathrm{Cl}$ interactions: orange dotted lines and $\mathrm{C}-\mathrm{H} \cdots \mathrm{Br}$ interactions: green dotted lines). (c) Slip-stacked arrangement of the CPMBP molecules along the [010] direction through $\pi \cdots \pi$ interactions. (d) Mechanically interlocked 3D network structures along the [010] plane. The blue arrow indicates the direction in which the mechanical force was applied during bending. (e) Optical microscope photographs of the brittle fracture during the three-point bending experiment of the single crystals of form I. 
(Fig. 2a and S5a $\dagger$ ). In the crystal structure, each molecule is connected to two other molecules via $\mathrm{C}-\mathrm{H} \cdots \mathrm{O}\left(2.62 \AA, 147.8^{\circ}\right)$ and $\mathrm{C}-\mathrm{H} \cdots \mathrm{Cl}\left(2.89 \AA, 146^{\circ}\right)$ interactions to form a corrugated ribbon (Fig. 2b). The molecules in the ribbon are slip-stacked along the crystallographic $b$-axis via $\pi \cdots \pi$ interactions (3.87 $\AA)$, which are further stabilized via $\mathrm{C}-\mathrm{H} \cdots \mathrm{Br}\left(2.95 \AA\right.$, $\left.148^{\circ}\right)$ interactions (Fig. 2c). The ribbons are connected via $\mathrm{C}-\mathrm{Cl} \cdots \pi$ interactions along the crystallographic $a$-axis. Together, these interactions lead to the formation of a mechanically interlocked 3D network structure, preventing the molecules from moving in response to mechanical stress (Fig. 2d). Hence, the crystal structure is consistent with the observed brittle fracture (Fig. 2e and S6a $\dagger$ ). ${ }^{16,44}$

Form II (plastic) crystallizes in the monoclinic space group $P 2_{1} / c$ with cell parameters $a=4.44 \AA, b=19.01 \AA, c=13.90 \AA$ and $\beta=90.18^{\circ}$ (Table $\mathrm{S} 1 \dagger$ ). Like in form $\mathrm{I}$, there is also one CPMBP molecule in the asymmetric unit that adopts a nearplanar conformation with a dihedral angle between the pyridyl and phenolic rings of $3.3^{\circ}$ due to the intramolecular $\mathrm{O}-\mathrm{H} \cdots \mathrm{N}$ hydrogen bonds (Fig. S4b; $\uparrow \mathrm{H} \cdots \mathrm{A}: 1.85 \AA$, $\theta$ : $146.2^{\circ}$ ). The two larger faces of the crystals of form II are the (010)/ $(0 \overline{1} 0)$ and $(001) /(00 \overline{1})$ faces, with the minor face being (100)/ (100) (Fig. 3a and S5b molecules are connected to dimers by the $\mathrm{C}-\mathrm{H} \cdots \mathrm{O}(2.64 \AA$, $148.4^{\circ}$ ) bonds (Fig. 3b). These dimers are slip-stacked via $\pi \cdots \pi$ interactions $(3.3 \AA$ ) along the growth axis of the crystal ( $a$-axis) to construct a columnar assembly (Fig. 3c). These stacked columns are close-packed in the orthogonal direction ( $b$-axis) through the weak hydrophobic -Cl groups (Fig. 3d). This feature provides slip planes or weak interaction planes along the [001] direction. The structure of form II is overall anisotropic with low energy slip planes, which is the conventional crystal engineering criterion for plastic bending (Fig. 3e and S6b†). Thus, upon mechanical stress perpendicular to the slip planes, the (010) face results in plastic deformation in the crystal of form II. $^{7}$ These low energy slip-planes may also contribute to the formation of extended defects which can influence mechanical properties. $^{68,69}$

The intermolecular interactions in both polymorphs were investigated via Hirshfeld surface analysis using Crystal Explorer 17.5. ${ }^{70}$ The contribution from all interactions in both the polymorphs is shown in Fig. $\mathrm{S} 7, \dagger$ which reveals the higher number of specific interactions in form I. Quantitative analysis of the intermolecular interactions in both polymorphic forms was conducted using the CoulombLondon-Pauli (CLP) PIXEL method for energy decomposition from quantum mechanical charge densities. ${ }^{63,64}$ This method has been widely applied to the study of intermolecular interactions in molecular solids. ${ }^{71-73}$ In brittle form I, the strongest interactions are those formed by $\pi \cdots \pi$ interactions along the $b$-axis $\left(-37.9 \mathrm{~kJ} \mathrm{~mol}^{-1}\right)$. In contrast, the interactions along the crystallographic $c$-axis (dominated by $\mathrm{C}-\mathrm{H} \cdots \mathrm{O}, \mathrm{C}-$ $\mathrm{H} \cdots \mathrm{Cl}$, and $\mathrm{C}-\mathrm{H} \cdots \mathrm{Br}$ interactions) average $c a .-27.5 \mathrm{~kJ} \mathrm{~mol}^{-1}$, with those along the crystallographic $a$-axis (comprising no specific interactions) being much weaker at ca. $-7.8 \mathrm{~kJ} \mathrm{~mol}^{-1}$. Consistent with prevailing design strategies for molecular flexibility, the intermolecular interaction energies for the plastic form II are more anisotropic than those for form I. The $\pi \cdots \pi$ interactions are somewhat stronger $(-40.7 \mathrm{~kJ}$ $\mathrm{mol}^{-1}$ ), reflecting the shorter interplanar spacing along the $\pi$ stacked axis. Interactions along the $c$-axis $(\mathrm{C}-\mathrm{H} \cdots \mathrm{O}$

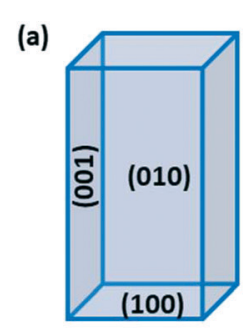

(b)

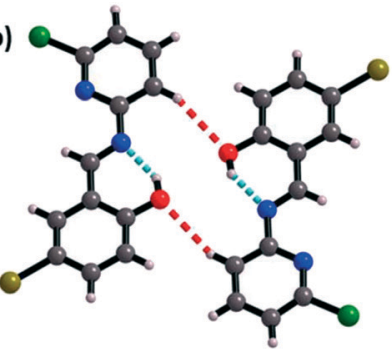

(d)

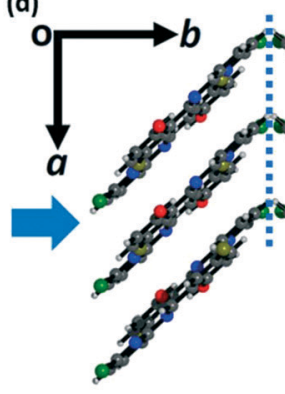

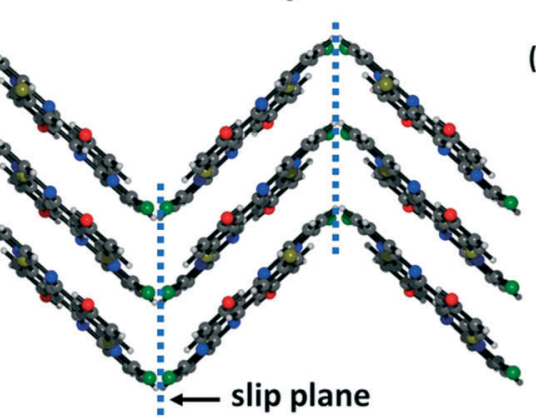

(c)

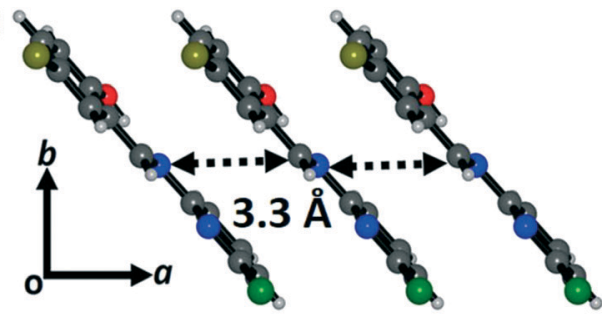

(e)

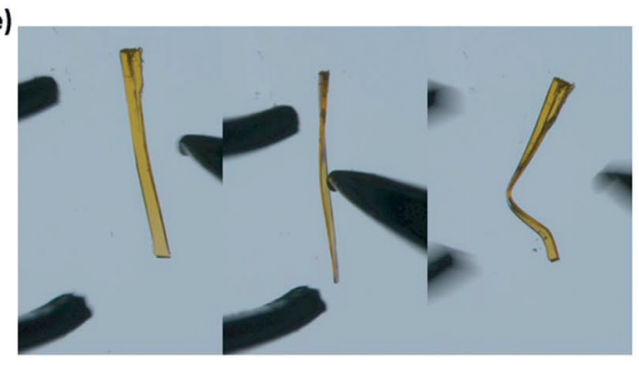

Fig. 3 Crystal packing of form II of CPMBP. (a) Schematic diagram of the crystal face indices determined by single-crystal X-ray diffraction. (b) The dimers are formed via $\mathrm{C}-\mathrm{H} \cdots \mathrm{O}$ interactions along the $\mathrm{c}$-axis $(\mathrm{C}-\mathrm{H} \cdots \mathrm{O}$ interactions: red dotted lines and $\mathrm{O}-\mathrm{H} \cdots \mathrm{N}$ interactions: cyan dotted lines). (c) Slip-stacked arrangement of the CPMBP molecules along the [001] direction through $\pi \cdots \pi$ interactions. (d) Molecular packing with slip planes along the (001) plane (cyan dotted lines). The blue arrow shows the direction in which the mechanical force is applied during bending. (e) Optical microscope photographs of the plastic bending upon mechanical stress along the (010) plane of the single crystals of form II. 
interactions) are again stronger, $-21.8 \mathrm{~kJ} \mathrm{~mol}^{-1}$, with the $b$-axis $\mathrm{C}-\mathrm{H} \cdots \pi$ interactions having energies of $c a .-12.8 \mathrm{~kJ}$ $\mathrm{mol}^{-1}$.

Following from the interesting effects of polymorphism on the mechanical properties, we sought to explore whether the polymorphism also affected the optical properties. To collect the solid-state photoluminescence (PL) spectra, the PL excitation (PLE) spectrum for each polymorph was first measured, Fig. S8. $\uparrow$ The respective maximum of each PLE spectrum was used as the excitation wavelength for the PL emission measurements. Both crystalline forms of CPMBP exhibited different emission properties, presumably owing to their distinct intermolecular interactions and molecular packing in the solid state, Fig. 5. A powdered sample of form I exhibited very weak orange emission at $605 \mathrm{~nm}\left(\lambda_{\mathrm{ex}}=425\right.$ $\mathrm{nm} ; \Phi=0.4 \%$ ) compared to powdered form II, which showed bright yellow emission at $585 \mathrm{~nm}\left(\lambda_{\mathrm{ex}}=425 \mathrm{~nm} ; \Phi=8.7 \%\right)$. The band gap for both forms is bound by predominantly carbon-based $2 \mathrm{p}_{z}$ states (see Fig. 6, S9 and S10 $\dagger$ ). We can therefore suggest that the red-shift of the form I emission spectrum (as compared with form II) presumably stems from its weaker $\pi-\pi$ stacking interactions (see Fig. 4).

The photoluminescence quantum yield,

$$
\Phi_{\mathrm{PL}}=\frac{k_{\mathrm{r}}}{k_{\mathrm{r}}+k_{\mathrm{nr}}}
$$

depends on the relative rate of radiative $k_{\mathrm{r}}$ and non-radiative $k_{\text {nr }}$ decay. ${ }^{74}$ In a crystal, the latter is dominated by emission of high-order phonons. ${ }^{75}$ As the vibrational spectra of both polymorphic forms are nearly identical, Fig. S3, $\uparrow$ we do not expect a significant difference in the non-radiative decay pathways between the polymorphic forms. We instead sought a qualitative rationale for the expected quantum yields by considering the differences in radiative decay rates. For

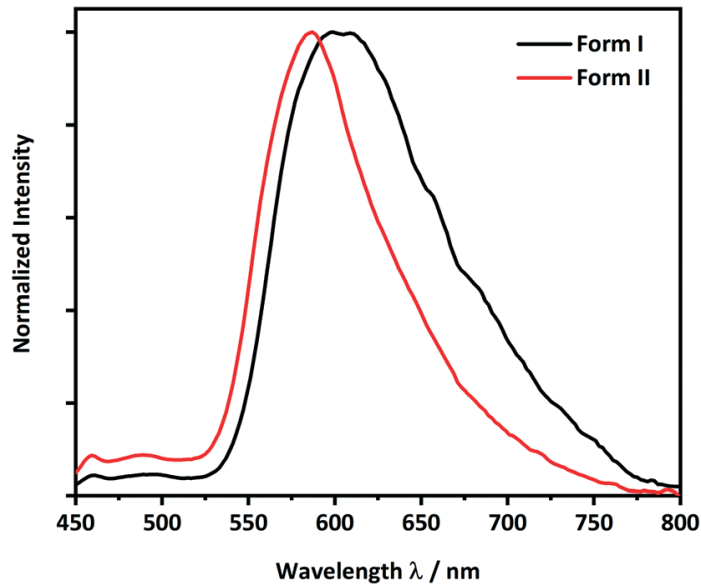

Fig. 5 Solid-state photoluminescence spectra of the brittle (form I, black line) and plastic (form II, red line) forms of CPMBP $\left(\lambda_{\mathrm{ex}}=425\right.$ $\mathrm{nm})$.

solids, $k_{\mathrm{r}}$ depends on whether the band gap is direct or indirect and on the probability of emission (here limited to spontaneous emission within the weak coupling regime). The band gaps calculated under periodic conditions at the PBEXDM level (see Fig. 6) are $1.82 \mathrm{eV}$ for form I (compared with the experimental $2.05 \mathrm{eV}$ optical gap) and $1.85 \mathrm{eV}$ for form II (compared with the experimental $2.12 \mathrm{eV}$ optical gap). Thus, our simulations are consistent with the experimentally observed trends in the photoluminescence emission wavelengths for both polymorphs, albeit with the expected underestimation from the use of a GGA functional. ${ }^{76}$ However, no indications of significant difference in the momentum transfer across the band gap were observed.

Under the highly idealized assumption that dipolar coupling is conserved in both polymorphic forms, decay can be roughly approximated with the number of available states
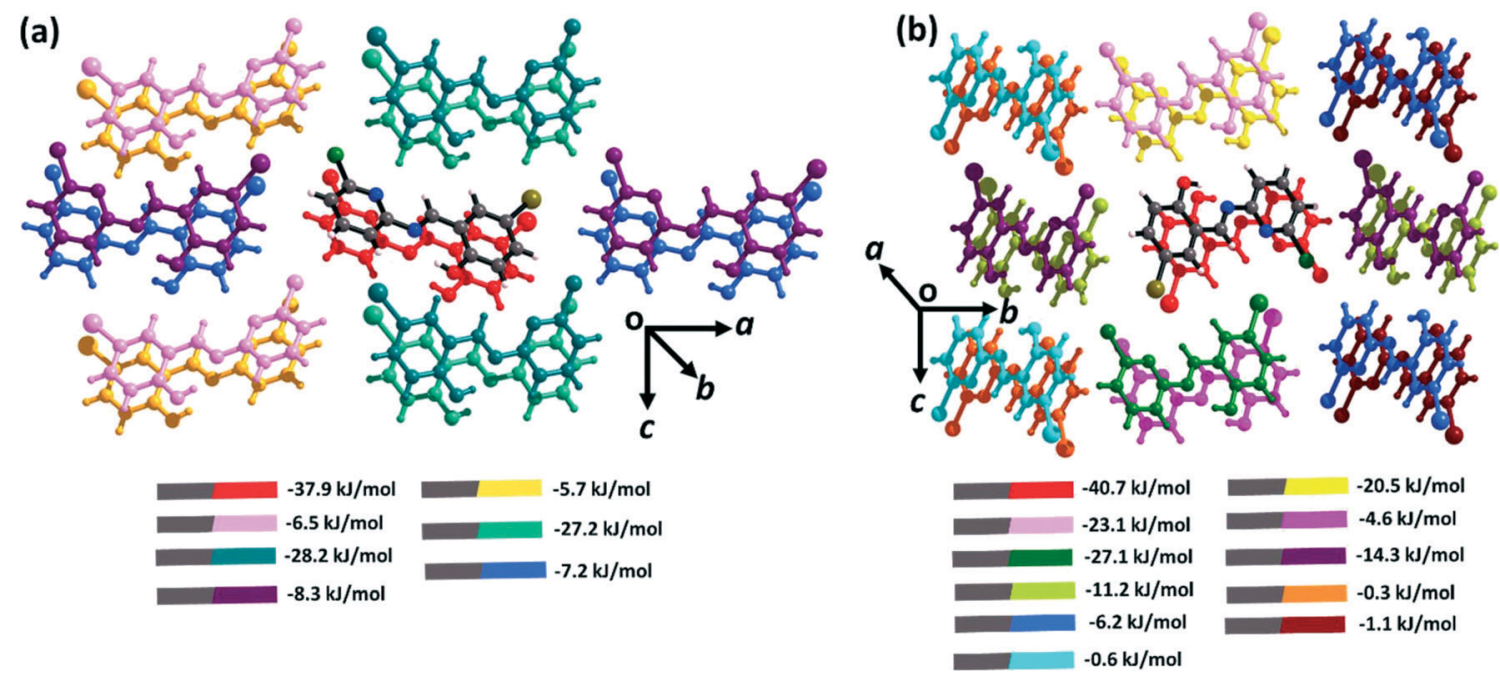

Fig. 4 Intermolecular interaction energies for (a) form I and (b) form II. All energies are described between the element-coloured molecule (denoted as grey in the legend) in the centre, and its coloured pair, as indicated in the legend. Full energy decomposition tables are provided in ESI† Tables S2-S4. 

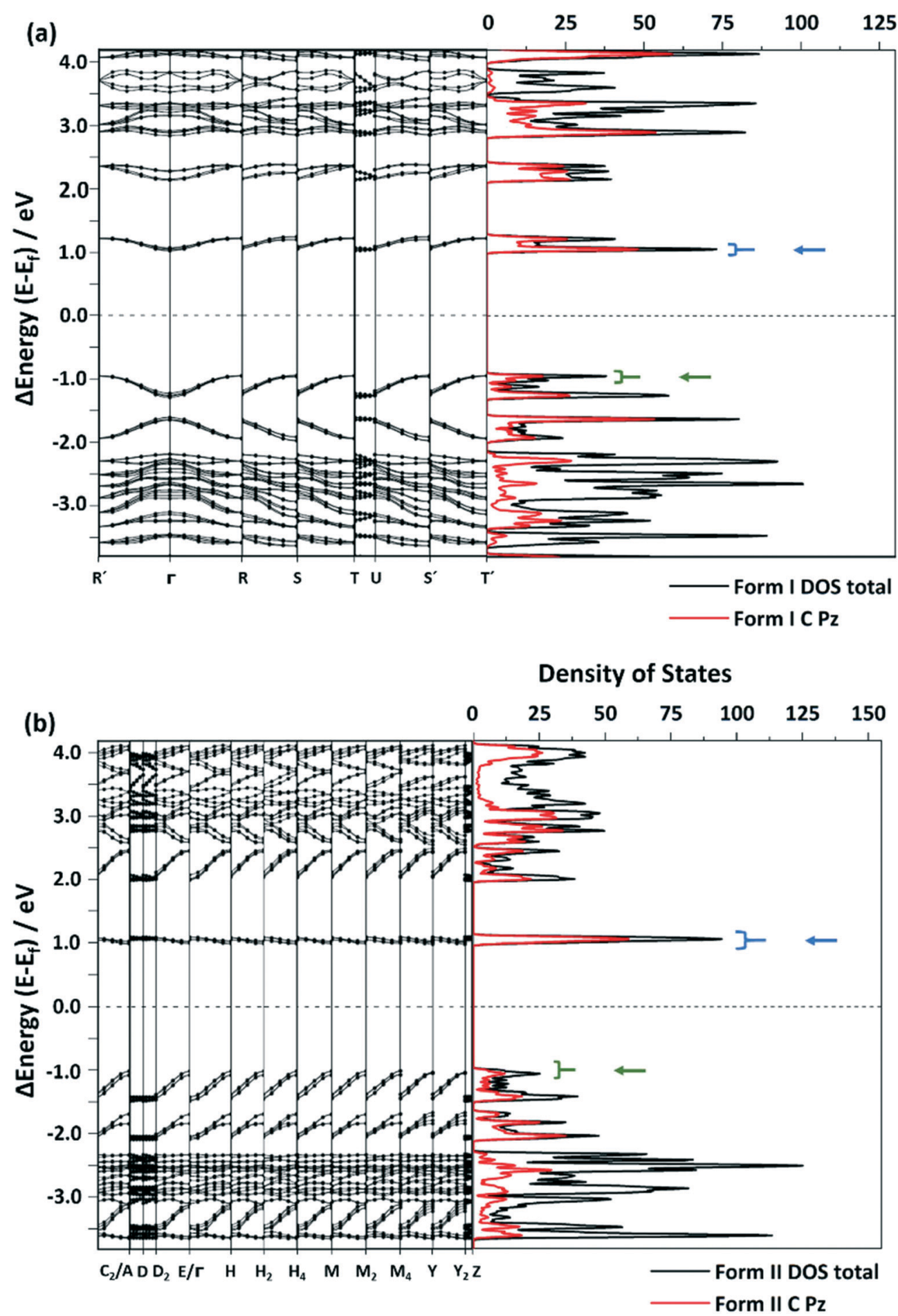

Fig. 6 Selected areas of the band structure and density of states (DOS) of (a) form I and (b) form II. The black line shows the total DOS and the red line represents the DOS projected onto $\mathrm{C}$-based atomic $2 \mathrm{p}_{z}$ orbitals. The blue arrow indicates the conduction band and the green arrow indicates the valence band. The complete band structure is provided in the ESI, $\uparrow$ Fig. S8 and S9.

in the conduction band $\left(\epsilon_{\mathrm{f}}\right)$ and the number of states in the valence band $\left(\epsilon_{\mathrm{i}}\right)$ via $k_{\mathrm{r}} \propto \int d \boldsymbol{k} \delta\left(\epsilon_{\mathrm{f}}(\boldsymbol{k})-\epsilon_{\mathrm{i}}(\boldsymbol{k})+\hbar \omega\right)$, where $\hbar \omega$ is the photon energy. By integrating over the corresponding states for each polymorphic form (Fig. 6), we observe $k_{\mathrm{r}}$ (form II) $>k_{\mathrm{r}}$ (form I), consistent with the experimental observations. Hence, stemming largely from the carbon-based $2 \mathrm{p}_{z}$ states, this further suggests that the different optical properties in the crystals stem from their unique $\pi \cdots \pi$ interactions. 
(a)

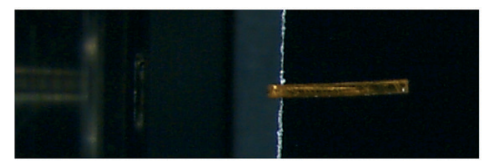

(b)

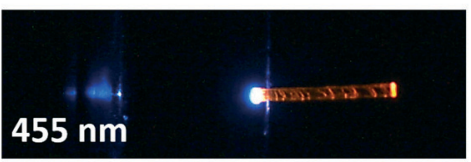

(c)

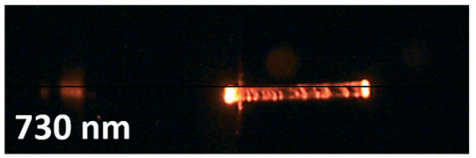

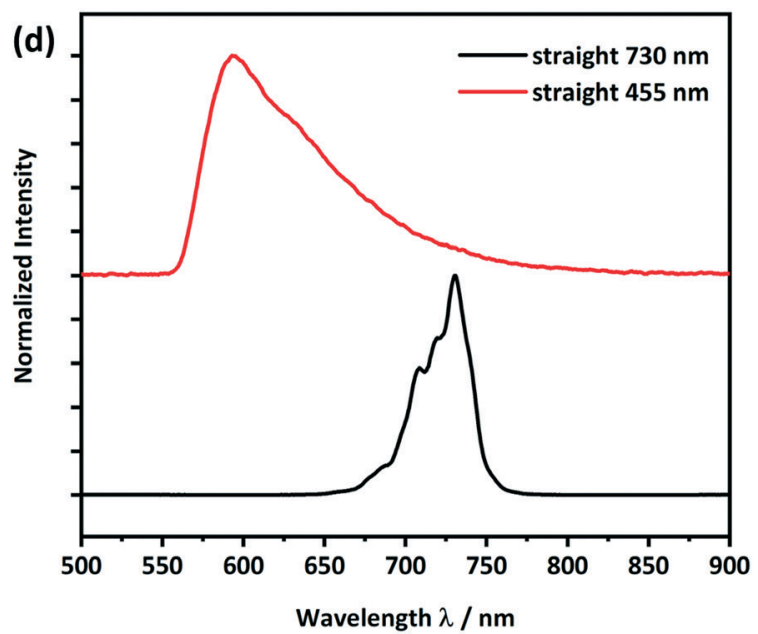

Fig. 7 Optical microscope images of a) a form II straight crystal placed on a silicon wafer with a $3 \mu \mathrm{m}$ thick $\mathrm{SiO}_{2}$ layer as shown in Scheme $\mathrm{S} 1, \uparrow$ b) showing light with a wavelength of $455 \mathrm{~nm}$ being coupled at the left end of the crystal and c) showing light with a wavelength of $730 \mathrm{~nm}$ being coupled at the left end of the crystal; d) detected PL spectra at the right end of the crystal. The spectra of the LED light sources, PLE and PL are given in Fig. S11 and S12.†

\section{Waveguiding properties}

To test the CPMBP single crystals as potential optical waveguides, incident light was focused through a microscope objective onto the (100) face of the crystals, Fig. 7. Emitted light was subsequently collected perpendicular to the optical axis of the waveguide. Two different LED photon sources were considered for incident light; a $455 \mathrm{~nm}$ source was used to test the active transport (i.e. where absorption and reemission are possible) while a $730 \mathrm{~nm}$ source was used to test for the passive transport (i.e. where absorption is not possible), Fig. 7b and c. In both cases, the guided light was observed to leak through defects along the crystal.

When using the $455 \mathrm{~nm}$ incident light source, the emission spectrum collected at the end of the crystal was very similar to the conventional solid-state PL spectrum (see Fig. 7d and 5). However, the high energy part of the guided emission spectrum is filtered out due to the selfabsorption of the CPMBP crystal. The ability of CPMBP to absorb its own photoluminescence on account of a small Stokes shift suggests it may be a promising candidate as a flexible wavelength-division multiplexing material. ${ }^{22,23,27}$ This effect will be the focus of follow-up investigation. In contrast, the guided emission spectrum from the $730 \mathrm{~nm}$ incident light source is unaffected by the crystal, Fig. $7 d$. The whole spectra of the used LED light sources, PLE and PL can be found in the ESI, $\dagger$ Fig. S11 (455 nm) and S12 (730 nm).

To observe the effect of plastic bending on the waveguiding properties of form II CPMBP, guided emission (a)

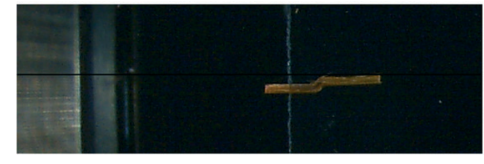

(b)

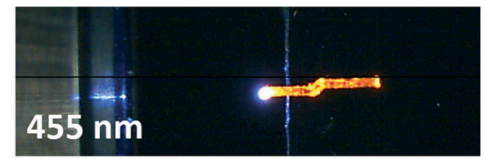

(c)

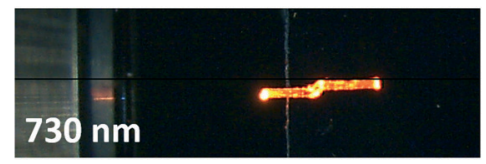

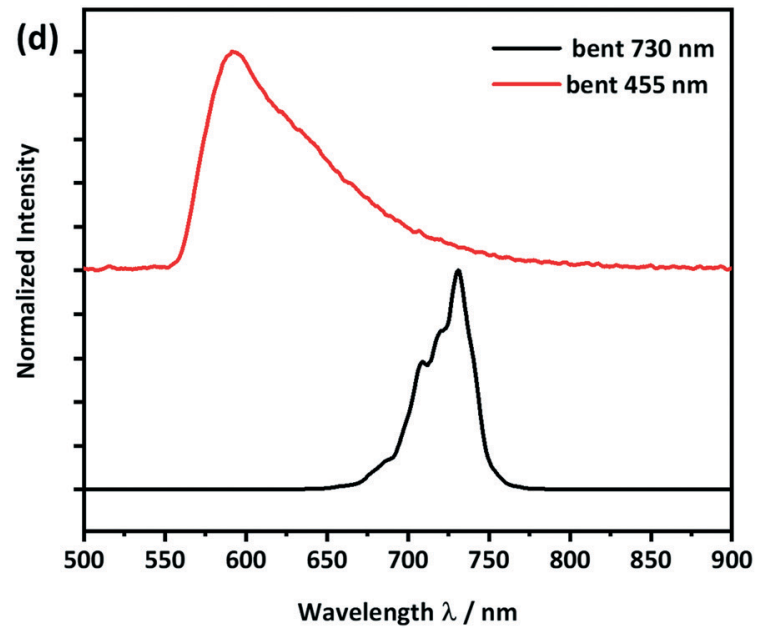

Fig. 8 Optical microscope images of (a) the bent crystal placed on a silicon wafer with a $3 \mu \mathrm{m}$ thick $\mathrm{SiO}_{2}$ layer, (b) showing light with a wavelength of $455 \mathrm{~nm}$ being coupled at the left end of the crystal and (c) showing light with a wavelength of $730 \mathrm{~nm}$ being coupled at the left end of the crystal; (d) detected PL-spectra at the right end of the crystal. 


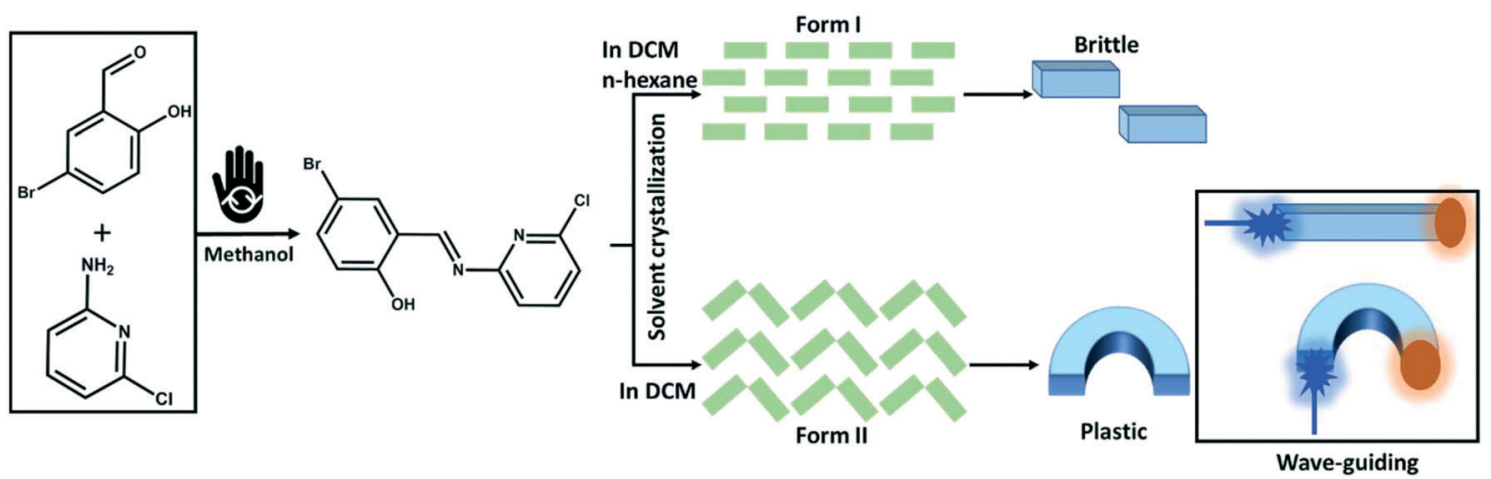

Scheme 1 Schematic representation of the polymorphic engineering of the mechanically flexible optical materials. The mechanochemical pictogram is taken from ref. 77.

spectra from both $455 \mathrm{~nm}$ and $730 \mathrm{~nm}$ incident light sources were again measured on the bent single crystals, Fig. 8. As compared with the straight crystals of form II (Fig. 7), no difference in the shape or intensity of the emission spectra at either incident energy was observed. This suggests that form II CPMBP conserves its waveguiding functionality upon deformation.

The promising optical behavior of CPMBP single crystals suggests significant technological potential. The combination of self-absorption and flexibility can be exploited to simplify light coupling into micron-scale waveguide systems. ${ }^{27,28}$ Since the CPMBP molecules form single crystals, it can be assumed that the structure affects the polarization of the propagating light. These characteristics of the CPMBP waveguide crystal can be applied in photonic systems for specific uses, which makes the CPMBP molecule an interesting material regarding photonic circuits.

\section{Conclusions}

We report here two polymorphic forms of a photoluminescent, 4-bromo-6-[(6-chloropyridin-2-ylimino) methyl]phenol with distinctly different optical and mechanical behaviors (Scheme 1). The two polymorphs showed a similar morphology and colour and could be obtained as pure forms by controlled crystallization using different solvent mixtures. Form I adopts a mechanically interlocked 3D network structure with various weak noncovalent interactions. In contrast, form II exhibits anisotropic crystal packing with low energy slip planes. Hence, form I was found to be mechanically brittle, whereas form II showed plastic deformation upon mechanical stress. Photoluminescence spectroscopy revealed that brittle form I is only weakly emissive whereas the emission of plastically flexible form II is comparatively bright. Only a minor red shift in the emission spectrum of form I is observed as compared with that of form II, which can be correlated with the stronger $\pi-\pi$ stacking interactions in the crystal packing of form I. Moreover, due to the shapable plastic flexibility combined with bright luminescence properties, form II exhibits the properties of bending the path of light, i.e., flexible optical waveguide. Both active and passive waveguiding were observed. We therefore demonstrate how the mechanical properties of organic luminophores can be tuned by controlling polymorphism instead of chemical modification. We expect polymorph engineering to become an important direction for designing mechanically flexible crystals with multifunctional applications in flexible optoelectronics.

\section{Conflicts of interest}

There are no conflicts to declare.

\section{Acknowledgements}

The authors thank BAM IT for access to computational resources. ELK and VS acknowledge the support from the Helmholtz Energy Materials Foundry (HEMF) and PEROSEED (ZT-0024) project. This work was carried out in the framework of the Joint Lab GEN_FAB. The authors are grateful to Prof Simon Parsons (University of Edinburgh) for access to the MrPIXEL software. BB acknowledges funding from Deutsche Forschungsgemeinschaft, Project No. 450137475.

\section{References}

1 Y. Su, G. Sui, J. Lan and X. Yang, Chem. Commun., 2020, 56, 11625-11628.

2 M. Wang, P. Baek, A. Akbarinejad, D. Barker and J. TravasSejdic, J. Mater. Chem. C, 2019, 7, 5534-5552.

3 H. R. Allcock, Soft Matter, 2012, 8, 7521-7532.

4 B. Kang, E. Song, S. B. Lee, B.-G. Chae, H. Ahn and K. Cho, Chem. Mater., 2018, 8, 6353-6360.

5 H. Sirringhaus, N. Tessler and R. H. Friend, Science, 1998, 280, 1741-1744.

6 P. Naumov, S. Chizhik, M. K. Panda, N. K. Nath and E. Boldyreva, Chem. Rev., 2015, 115, 12440-12490.

7 G. R. Krishna, R. Devarapalli, G. Lal and C. M. Reddy, J. Am. Chem. Soc., 2016, 138, 13561-13567.

8 S. Ghosh and C. M. Reddy, Angew. Chem., 2012, 5.

9 S. Ghosh, M. K. Mishra, S. B. Kadambi, U. Ramamurty and G. R. Desiraju, Angew. Chem., 2015, 127, 2712-2716. 
10 N. P. Thekkeppat, M. Lakshmipathi, A. S. Jalilov, P. Das, A. M. P. Peedikakkal and S. Ghosh, Cryst. Growth Des., 2020, 20, 3937-3943.

11 P. Gupta, T. Panda, S. Allu, S. Borah, A. Baishya, A. Gunnam, A. Nangia, P. Naumov and N. K. Nath, Cryst. Growth Des., 2019, 19, 3039-3044.

12 P. Gupta, D. P. Karothu, E. Ahmed, P. Naumov and N. K. Nath, Angew. Chem., Int. Ed., 2018, 5.

13 S. Hayashi, T. Koizumi and N. Kamiya, Cryst. Growth Des., 2017, 17, 6158-6162.

14 E. Ahmed, D. P. Karothu and P. Naumov, Angew. Chem., Int. Ed., 2018, 10.

15 S. Saha, M. K. Mishra, C. M. Reddy and G. R. Desiraju, Acc. Chem. Res., 2018, 51, 2957-2967.

16 C. M. Reddy, G. Rama Krishna and S. Ghosh, CrystEngComm, 2010, 12, 2296.

17 B. Bhattacharya, A. A. L. Michalchuk, D. Silbernagl, M. Rautenberg, T. Schmid, T. Feiler, K. Reimann, A. Ghalgaoui, H. Sturm, B. Paulus and F. Emmerling, Angew. Chem., Int. Ed., 2020, 59, 5557-5561.

18 M. Đaković, M. Borovina, M. Pisačić, C. B. Aakeröy, Ž. Soldin, B.-M. Kukovec and I. Kodrin, Angew. Chem., Int. Ed., 2018, 57, 14801-14805.

19 L. Mei, S. An, K. Hu, L. Wang, J. Yu, Z. Huang, X. Kong, C. Xia, Z. Chai and W. Shi, Angew. Chem., 2020, 8.

20 N. K. Nath, P. Gupta, P. J. Hazarika, N. Deka, A. Mukherjee and G. K. Dutta, Cryst. Growth Des., 2019, 19, 6033-6038.

21 H. Liu, Z. Bian, Q. Cheng, L. Lan, Y. Wang and H. Zhang, Chem. Sci., 2019, 10, 227-232.

22 H. Liu, Z. Lu, Z. Zhang, Y. Wang and H. Zhang, Angew. Chem., Int. Ed., 2018, 57, 8448-8452.

23 H. Liu, Z. Bian, Q. Cheng, L. Lan, Y. Wang and H. Zhang, Chem. Sci., 2019, 10, 227-232.

24 L. Catalano, D. P. Karothu, S. Schramm, E. Ahmed, R. Rezgui, T. J. Barber, A. Famulari and P. Naumov, Angew. Chem., Int. Ed., 2018, 57, 17254-17258.

25 S. Hayashi, S. Yamamoto, D. Takeuchi, Y. Ie and K. Takagi, Angew. Chem., Int. Ed., 2018, 57, 17002-17008.

26 J. Cao, H. Liu and H. Zhang, CCS Chem., 2020, 2, 2569-2575.

27 M. Annadhasan, A. R. Agrawal, S. Bhunia, V. V. Pradeep, S. S. Zade, C. M. Reddy and R. Chandrasekar, Angew. Chem., Int. Ed., 2020, 59, 13852-13858.

28 M. Annadhasan, D. P. Karothu, R. Chinnasamy, L. Catalano, E. Ahmed, S. Ghosh, P. Naumov and R. Chandrasekar, Angew. Chem., Int. Ed., 2020, 59, 13821-13830.

29 B. Bhattacharya, D. Roy, S. Dey, A. Puthuvakkal, S. Bhunia, S. Mondal, R. Chowdhury, M. Bhattacharya, M. Mandal, K. Manoj, P. K. Mandal and C. M. Reddy, Angew. Chem., 2020, 6.

30 S. Hayashi, F. Ishiwari, T. Fukushima, S. Mikage, Y. Imamura, M. Tashiro and M. Katouda, Angew. Chem., Int. Ed., 2020, 7.

31 E. M. Horstman, R. K. Keswani, B. A. Frey, P. M. Rzeczycki, V. LaLone, J. A. Bertke, P. J. A. Kenis and G. R. Rosania, Angew. Chem., Int. Ed., 2017, 56, 1815-1819.
32 L. Adler-Abramovich, Z. A. Arnon, X. Sui, I. Azuri, H. Cohen, O. Hod, L. Kronik, L. J. W. Shimon, H. D. Wagner and E. Gazit, Adv. Mater., 2018, 30, 1704551.

33 S. Ghosh and M. K. Mishra, Cryst. Growth Des., 2021, 21, 2566-2580.

34 P. Gupta, S. A. Rather, B. K. Saha, T. Panda, D. P. Karothu and N. K. Nath, Cryst. Growth Des., 2020, 6.

35 N. K. Nath, M. Hazarika, P. Gupta, N. R. Ray, A. K. Paul and E. Nauha, J. Mol. Struct., 2018, 1160, 20-25.

36 U. B. R. Khandavilli, M. Lusi and P. J. Frawley, IUCrJ, 2019, 6, 630-634.

37 S. Saha and G. R. Desiraju, J. Am. Chem. Soc., 2018, 13.

38 S. Saha and G. R. Desiraju, Chem. Commun., 2016, 52, 7676-7679.

39 B. Puschner, R. H. Poppenga, L. J. Lowenstine, M. S. Filigenzi and P. A. Pesavento, J. Vet. Diagn. Invest., 2007, 19, 616-624.

40 A. J. Cruz-Cabeza and J. Bernstein, Chem. Rev., 2014, 114, 2170-2191.

41 C. M. Reddy, S. Basavoju and G. R. Desiraju, Chem. Commun., 2005, 2439-2441.

42 S. Saha and G. R. Desiraju, Chem. Commun., 2018, 54, 6348-6351.

43 M. K. Mishra and C. C. Sun, Cryst. Growth Des., 2020, 20, 4764-4769.

44 K. B. Raju, S. Ranjan, V. S. Vishnu, M. Bhattacharya, B. Bhattacharya, A. K. Mukhopadhyay and C. M. Reddy, Cryst. Growth Des., 2018, 18, 3927-3937.

45 X. Chu, Z. Lu, B. Tang, B. Liu, K. Ye and H. Zhang, J. Phys. Chem. Lett., 2020, 11, 5433-5438.

46 B. Liu, H. Liu, H. Zhang, Q. Di and H. Zhang, J. Phys. Chem. Lett., 2020, 11, 9178-9183.

47 N. Mitetelo, D. Venkatakrishnarao, J. Ravi, M. Popov, E. Mamonov, T. V. Murzina and R. Chandrasekar, Adv. Opt. Mater., 2019, 7, 1801775.

48 W. Zhang, J. Yao and Y. S. Zhao, Acc. Chem. Res., 2016, 49, 1691-1700.

49 K. Takazawa, J. Inoue, K. Mitsuishi and T. Kuroda, Adv. Funct. Mater., 2013, 23, 839-845.

50 V. Coropceanu, J. Cornil and J.-L. Bredas, Chem. Rev., 2007, 107, 926-952.

51 H. Zhang, H. Liu, Z. Lu, B. Tang, C. Qu and Z. Zhang, Angew. Chem., 2020, 132, 13044-13050.

52 M. Mathivanan, B. Tharmalingam, C.-H. Lin, B. V. Pandiyan, V. Thiagarajan and B. Murugesapandian, CrystEngComm, 2020, 22, 213-228.

53 G. M. Sheldrick, Acta Crystallogr., Sect. A: Found. Crystallogr., 2008, 64, 112-122.

54 G. Sheldrick, SADABS, University of Göttingen, Germany, 2002.

55 G. M. Sheldrick, Acta Crystallogr., Sect. A: Found. Adv., 2015, 71, 3-8.

56 G. M. Sheldrick, Acta Crystallogr., Sect. C: Struct. Chem., 2015, 71, 3-8.

57 P. Giannozzi, S. Baroni, N. Bonini, M. Calandra, R. Car, C. Cavazzoni, D. Ceresoli, G. L. Chiarotti, M. Cococcioni, I. Dabo, A. Dal Corso, S. de Gironcoli, S. Fabris, G. Fratesi, R. 
Gebauer, U. Gerstmann, C. Gougoussis, A. Kokalj, M. Lazzeri, L. Martin-Samos, N. Marzari, F. Mauri, R. Mazzarello, S. Paolini, A. Pasquarello, L. Paulatto, C. Sbraccia, S. Scandolo, G. Sclauzero, A. P. Seitsonen, A. Smogunov, P. Umari and R. M. Wentzcovitch, J. Phys.: Condens. Matter, 2009, 21, 395502.

58 P. Giannozzi, O. Andreussi, T. Brumme, O. Bunau, M. Buongiorno Nardelli, M. Calandra, R. Car, C. Cavazzoni, D. Ceresoli, M. Cococcioni, N. Colonna, I. Carnimeo, A. Dal Corso, S. de Gironcoli, P. Delugas, R. A. DiStasio, A. Ferretti, A. Floris, G. Fratesi, G. Fugallo, R. Gebauer, U. Gerstmann, F. Giustino, T. Gorni, J. Jia, M. Kawamura, H.-Y. Ko, A. Kokalj, E. Küçükbenli, M. Lazzeri, M. Marsili, N. Marzari, F. Mauri, N. L. Nguyen, H.-V. Nguyen, A. Otero-de-la-Roza, L. Paulatto, S. Poncé, D. Rocca, R. Sabatini, B. Santra, M. Schlipf, A. P. Seitsonen, A. Smogunov, I. Timrov, T. Thonhauser, P. Umari, N. Vast, X. Wu and S. Baroni, J. Phys.: Condens. Matter, 2017, 29, 465901.

59 J. P. Perdew, K. Burke and M. Ernzerhof, Phys. Rev. Lett., 1996, 77, 3865-3868.

60 A. D. Becke and E. R. Johnson, J. Chem. Phys., 2005, 122, 154104.

61 P. E. Blöchl, Phys. Rev. B: Condens. Matter Mater. Phys., 1994, 50, 17953-17979.

62 H. J. Monkhorst and J. D. Pack, Phys. Rev. B: Solid State, 1976, 13, 5188-5192.

63 A. Gavezzotti, New J. Chem., 2011, 1360-1368.

64 A. Gavezzotti, Mol. Phys., 2008, 106, 1473-1485.

65 M. G. Reeves, P. A. Wood and S. Parsons, J. Appl. Crystallogr., 2020, 53, 1154-1162.

66 M. J. Frisch, G. W. Trucks, H. B. Schlegel, G. E. Scuseria, M. A. Robb, J. R. Cheeseman, G. Scalmani, V. Barone, G. A. Petersson, H. Nakatsuji, X. Li, M. Caricato, A. V. Marenich, J. Bloino, B. G. Janesko, R. Gomperts, B. Mennucci, H. P. Hratchian, J. V. Ortiz, A. F. Izmaylov, J. L. Sonnenberg, D. Williams-Young, F. Ding, F. Lipparini, F. Egidi, J. Goings, B.
Peng, A. Petrone, T. Henderson, D. Ranasinghe, V. G. Zakrzewski, J. Gao, N. Rega, G. Zheng, W. Liang, M. Hada, M. Ehara, K. Toyota, R. Fukuda, J. Hasegawa, M. Ishida, T. Nakajima, Y. Honda, O. Kitao, H. Nakai, T. Vreven, K. Throssell, J. A. Montgomery Jr., J. E. Peralta, F. Ogliaro, M. J. Bearpark, J. J. Heyd, E. N. Brothers, K. N. Kudin, V. N. Staroverov, T. A. Keith, R. Kobayashi, J. Normand, K. Raghavachari, A. P. Rendell, J. C. Burant, S. S. Iyengar, J. Tomasi, M. Cossi, J. M. Millam, M. Klene, C. Adamo, R. Cammi, J. W. Ochterski, R. L. Martin, K. Morokuma, O. Farkas, J. B. Foresman and D. J. Fox, Gaussian 16 Revision A.01, 2016.

67 G. Socrates, Infrared and Raman Characteristic Group Frequencies: Tables and Charts, Wiley, New York, 2001.

68 I. A. Olson, A. G. Shtukenberg, B. Kahr and M. D. Ward, Rep. Prog. Phys., 2018, 81, 096501.

69 M. M. Kuklja and A. B. Kunz, J. Appl. Phys., 2001, 89, 10.

70 P. R. Spackman, M. J. Turner, J. J. McKinnon, S. K. Wolff, D. J. Grimwood, D. Jayatilaka and M. A. Spackman, J. Appl. Crystallogr., 2021, 54, 1-6.

71 F. P. A. Fabbiani, D. R. Allan, S. Parsons and C. R. Pulham, CrystEngComm, 2005, 7, 179.

72 N. P. Funnell, C. L. Bull, C. J. Ridley, S. Parsons and J. P. Tellam, Chem. Commun., 2020, 56, 6428-6431.

73 N. Giordano, C. M. Beavers, K. V. Kamenev, W. G. Marshall, S. A. Moggach, S. D. Patterson, S. J. Teat, J. E. Warren, P. A. Wood and S. Parsons, CrystEngComm, 2019, 21, 4444-4456.

74 C. Deng, Y. Niu, Q. Peng, A. Qin, Z. Shuai and B. Z. Tang, J. Chem. Phys., 2011, 135, 014304.

75 J. Collins, ECS J. Solid State Sci. Technol., 2016, 5, R3170-R3184.

76 J. M. Crowley, J. Tahir-Kheli and W. A. Goddard, J. Phys. Chem. Lett., 2016, 7, 1198-1203.

77 A. A. L. Michalchuk, E. V. Boldyreva, A. M. Belenguer, F. Emmerling and V. V. Boldyrev, Front. Chem., 2021, 9, 685789. 